\title{
Lebed's resonance in organic conductors in the vicinity of field induced spin density wave transition
}

\author{
Y. SUN and K. MAKI \\ Department of Physics and Astronomy, University of Southern California, Los Angeles, CA 90089-0484, \\ U.S.A.
}

\begin{abstract}
We study both the stable FISDW and the fluctuation contribution near the Lebed resonance observed in (TMTSF) ${ }_{2} \mathrm{X}$ with $\mathrm{X}=\mathrm{ClO}_{4}$ and $\mathrm{PF}_{6}$ (under pressure). We find the most stable state at the Lebed resonance (we call it the Lebed State) is charaterized by a pair of integers $(\mathrm{N}, \mathrm{M})$; Both $\sigma_{a b}$ and $\sigma_{a c}$ are quantized where $a$ means the best conducting direction. Also numerical analysis indicates the SDW fluctuation gives rise to peaks in $\sigma_{a a}$ at the Lebed resonances consistent with experiments.
\end{abstract}

\section{Introduction.}

Since a remarkable prediction by Lebed [1] that there should be resonance-like effect in the bulk properties of quasi-one dimensional conductors with open Fermi surfaces when a magnetic field in the plane perpendicular to the best conducting direction (i.e. the $a$ axis) but tilted from the least conducting direction (the $c^{*}$ axis) by definite angles (which we shall call the Lebed angle), a number of experiments observed dips in the magnetoresistance at the predicted angles [2-4] in Bechgaard salts at low temperatures. On the other hand the predicted instability in the normal state has never been observed $[5,6]$. In fact Chen and one of authors [7] have shown that the Lebed resonance stabilizes the normal state by suppressing the FISDW transition.

As to the origin of dips in the magnetoresistance two models have been proposed: the scattering model $[8,9]$ and the SDW fluctuation model [10], since all other models [11, 12] give rather small effects. However, the scattering model by Lebed and Bak [8] gave dips in the conductivity at the Lebed resonance. On the other hand the fluctuation model proposed by Bjelis and Maki [10] is shown to give peaks at the resonance. The fluctuation contribution to the conductivity is given by

$$
\delta \sigma_{a a}=-\frac{1}{4} e^{2} \alpha \xi_{a}\left(\xi_{b} \xi_{c}\right)^{-1}(\sqrt{\eta}+\sqrt{\delta})^{-1}
$$

where $\alpha$ is a new kinetic coefficient of order of unity so far neglected, $\xi_{a}, \xi_{b}$ and $\xi_{c}$ are the temperature independent coherence lengths in the $a, b$ and $c$ directions and $\eta=\ln \left(T / T_{c}(B, \theta)\right)$ with $T_{c}(B, \theta)$ is the FISDW transition temperature. $\delta \cong\left(\xi_{a} / l_{a}\right)^{2}$ is the dimensionless pair-breaking parameter and $l_{a}$ is the electron mean free path in the $a$ direction. For a typical pristine Bechgaard crystal sample we expect $l_{a} / \xi_{a} \cong 10$. In [10] 
it was assumed that only $\xi_{c}$ is very sensitive to the presence of the Lebed resonance. As we shall see all of them $\alpha, \xi_{a}, \xi_{b}, \xi_{c}$ and $T_{c}$ develop sharp dips at the Lebed resonance. Therefore indeed Eq(1) predicts peaks at the Lebed resonance consistent with experiments [2 4].

Extending an early analysis [7] we explore the stable FISDW at the Lebed resonance. We find that the FISDW at the resonance is characterized by a pair of integers $(N, M)$. These states have doubly quantized Hall effect in both the $a-b$ plane and the $a-c$ plane. The Hall conductivities are $\sigma_{a b}=2 e^{2} \mathrm{~N} / \mathrm{ch}$ and $\sigma_{a c}=2 e^{2} M / b h$, which can be easily deduced from Yakovenko's theory [13].

\section{Fluctuation Propagator.}

As in [10] we consider an anisotropic Hubbard model (i.e. Yamaji's model [14]) in a magnetic field perpendicular to the most conducting direction. The SDW fluctuation propagator is given within mean field theory

$D^{-1}\left(\vec{q}, \omega_{v}\right)=N_{o}\left\{\ln \frac{T}{T_{c}}+\xi_{a}^{2} q_{1}^{2}+\xi_{b}^{2} q_{2}^{2}+\xi_{c}^{2} q_{3}^{2}+\frac{\alpha \pi}{8 T}\left|\omega_{v}\right|\right\}$

where $N_{o}=(\pi v b c)^{-1}$ is the electron density of states per spin at the Fermi Surface, $T$ is the temperature, $\omega_{v}$ is the Matsubara frequency and the FISDW transition temperature $T_{c}$ is given by

$-\ln \left(\frac{T_{c}}{T_{c o}}\right)=\sum_{n, m}\left|I_{n m}\right|^{2}\left\{\operatorname{Re} \Psi\left(\frac{1}{2}+i x_{n m}\right)-\Psi\left(\frac{1}{2}\right)\right\}$

and

$x_{n m}=\left[(n-N) \omega_{b}-(m-M) \omega_{c}\right] / 4 \pi T_{c}$

$\omega_{b}=v b e B \cos \theta, \quad \omega_{c}=v c e B \sin \theta$

and

$I_{n m}=\sum_{l_{1} l_{2} l_{3}}(i)^{l_{2}+l_{3}} J_{l_{1}}(\alpha) J_{l_{2}}(\beta) J_{l_{3}}(\gamma) J_{\frac{1}{2}\left(n-m-l_{2}-l_{3}\right)-l_{1}}\left(\gamma_{1}\right) J_{\frac{1}{2}\left(n+m-l_{2}+l_{3}\right)-l_{1}}\left(\gamma_{2}\right)$

and

$$
\begin{gathered}
\alpha=\varepsilon_{0} \cos \phi / \omega_{b}, \beta=4 t_{b} \sin \left(\frac{1}{2} \phi\right) / \omega_{b} \\
\gamma=-4 t_{c} \sin \left(\frac{1}{2} \phi^{\prime}\right) / \omega_{c}, \gamma_{1}=2 \varepsilon_{1} \cos \left(\frac{1}{2}\left(\phi+\phi^{\prime}\right)\right)\left(\omega_{b}-\omega_{c}\right)^{-1} \\
\gamma_{2}=2 \varepsilon_{1} \cos \left(\frac{1}{2}\left(\phi-\phi^{\prime}\right)\right)\left(\omega_{b}+\omega_{c}\right)^{-1} \\
\varepsilon_{0}=t_{b}^{2} / \sqrt{2} t_{a}, \varepsilon_{1}=\sqrt{2} t_{b} t_{c} / t_{a}
\end{gathered}
$$

and $t_{a}, t_{b}$ and $t_{c}$ are the electron transfer integrals in the $a, b$ and $c$ directions. For Bechgaard salts we take typical values $t_{a}=2992 \mathrm{~K}, t_{b}=299 \mathrm{~K}$ and $t_{c}=10 \mathrm{~K}[15]$. The integers $N, M$ and angles $\phi$ and $\phi$ have to be determined to maximize $T_{c}(B, \theta)$. Here $\Psi(z)$ is the di-gamma function, $J_{l}(\mathrm{z})$ is the Bessel function and $T_{c 0}$ is the SDW transition temperature for $\varepsilon_{0}=0$ and in the absence of magnetic field. The state with $M=0$ 
corresponds to the $N$ th FISDW well known already [16-18]. Except near the Lebed resonance $\tan \theta=b / c$ (i.e. $\left.\theta \cong 30^{\circ}\right), I_{n m}$ in $\mathrm{Eq}(5)$ is well approximated by

$$
I_{n m}=\sum_{l_{1} l_{2}}(i)^{l_{2}} J_{l_{1}}(\alpha) J_{l_{2}}(\beta) J_{\frac{1}{2}\left(n-m-l_{2}\right)-l_{1}}\left(\gamma_{1}\right) J_{\frac{1}{2}\left(n+m-l_{2}\right)-l_{1}}\left(\gamma_{2}\right)
$$

while in the vicinity of the resonance we have

$$
I_{n m}=(i)^{n-m} J_{m}(\gamma) \sum_{l}(-1)^{l} J_{l}(\alpha) J_{n-2 l}(\beta)
$$

In $\mathrm{Eq}(7)$ we took $\phi=0$ in order to eliminate the $\gamma$ term, while in $\mathrm{Eq}(8) \phi+\phi=\pi$ to eliminate the $\gamma_{1}$ term from $\mathrm{Eq}(5)$. As we shall see the latter choice makes the $M \neq 0$ state more favorable implying the quantum Hall effect in the $a-c$ plane as well.

The coherence lengths $\xi_{a}, \xi_{b}$, and $\xi_{c}$ are similarly obtained as [10];

$$
\begin{aligned}
& \xi_{a}^{2}=-\frac{v^{2}}{2\left(4 \pi T_{c}\right)^{2}} \sum_{n, m}\left|I_{n m}\right|^{2} \operatorname{Re} \Psi^{(2)}\left(\frac{1}{2}+i x_{n m}\right) \\
& \xi_{b}^{2}=\frac{v_{b}{ }^{2} x_{1}^{-2}}{2\left(4 \pi T_{c}\right)^{2}} \sum_{n m}\left|I_{n m}\right|^{2} \operatorname{Re}\left\{\Psi\left(\frac{1}{2}+i x_{n+1, m}\right)+\Psi\left(\frac{1}{2}+i x_{n-1, m}\right)-2 \Psi\left(\frac{1}{2}+i x_{n m}\right)\right\} \\
& \xi_{c}^{2}=\frac{v_{c}{ }^{2} x_{2}^{-2}}{2\left(4 \pi T_{c}\right)^{2}} \sum_{n m}\left|I_{n m}\right|^{2} \operatorname{Re}\left\{\Psi\left(\frac{1}{2}+i x_{n, m+1}\right)+\Psi\left(\frac{1}{2}+i x_{n, m-1}\right)-2 \Psi\left(\frac{1}{2}+i x_{n m}\right)\right\}
\end{aligned}
$$

and $x_{1}=\omega_{b} / 4 \pi T_{c}$ and $x_{2}=\omega_{c} / 4 \pi T_{c}$.

Finally $\alpha$ is given by

$$
\alpha=\sum_{n m}\left|I_{n m}\right|^{2} \operatorname{sech}^{2}\left(\pi x_{n m}\right)
$$

In Fig. 1 we show the FISDW transition temperature $T_{c}$ as function of $\theta$, the tilt angle (i.e. the angle $\vec{B}$ makes from the $c^{*}$ axis) for $\omega_{b}(\theta=0) \Delta_{0}=1$. For Bechgaard salts we take $T_{c 0}=12 \mathrm{~K}$ and $\Delta_{0}=21 \mathrm{~K}$ and $\omega_{b}(\theta=0)=$ $\Delta_{0}$ corresponds roughly to 30 Tesla. We take $\varepsilon_{0}=\Delta_{0}$ in the present analysis which approximate (TMTSF) 2 $\mathrm{PF}_{6}$ under pressure $(\sim 7 k$ bar). From Fig 1 we see clearly the $(0,0)$ at $\theta=0$ develop a dip at the Lebed resonance $\left(\theta \cong 30^{\circ}\right)$. At the resonance this state is replaced by the $(0,1)$ state. Beyond the resonance we recover the initial states $(0,0)$ which is ultimately replaced by $(1,0)$ state at larger $\theta$. More generally the FISDW at the Lebed resonance is doubly quantized; the Hall conductivities both in the $a-b$ plane and the $a$ $c$ plane are quantized. We obtain

$$
\sigma_{a b}=2 e^{2} N / c h \quad, \quad \sigma_{a c}=2 e^{2} M / b h
$$



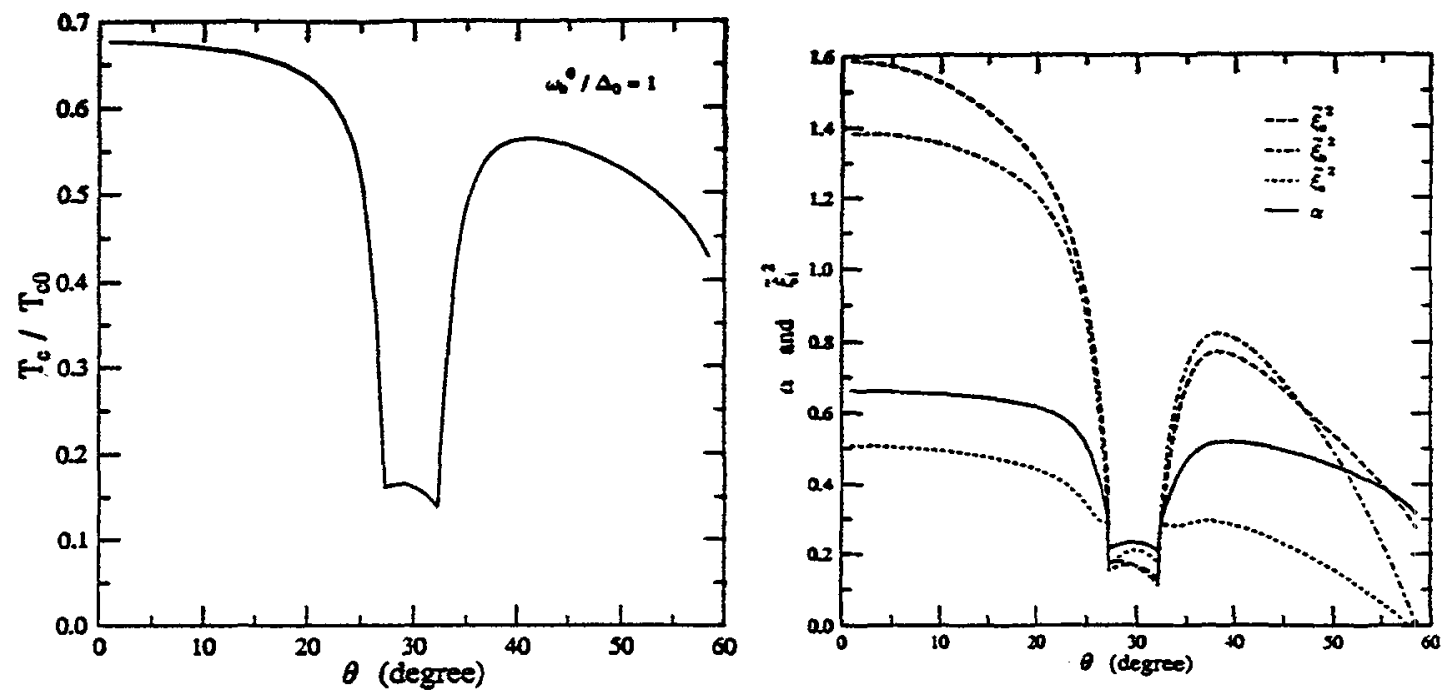

Fig. 1. - The SDW transition temperature $T_{c}$ is shown as function of $\theta$ for $\omega_{b}{ }^{0} / \Delta_{0}=1$.

Fig. 2. $-\alpha$ and $\tilde{\xi}_{i}^{2}$ are shown as function of $\theta$ for $\omega_{b}{ }^{0} / \Delta_{0}=1$
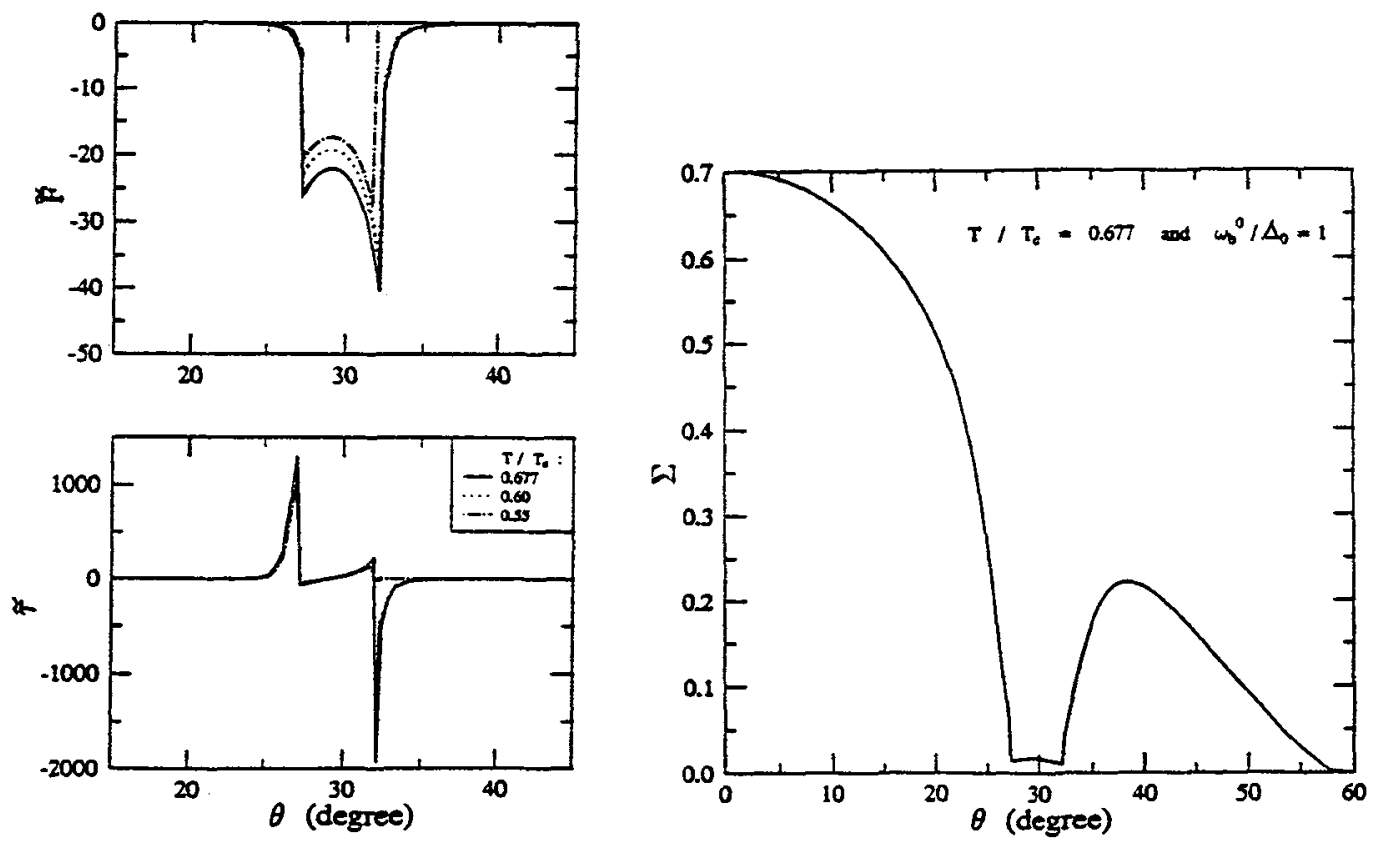

Fig. 3. $--\tilde{F}$ and $\tilde{\tau}$ are shown as function of $\theta$ for $T / T_{c}=0.677,0.60$ and 0.55 .

Fig. 4. $-\sum$ is shown as function of $\theta$ for $\omega_{b} \% / \Delta_{0}=1$. 
where $h$ is the Planck constant. Here we made use of the theory developed by Yakovenko [13].

In Figs. 2 we show corresponding $\tilde{\xi}_{a}^{2}, \tilde{\xi}_{b}^{2}, \tilde{\xi}_{c}^{2}$ and $\alpha$ as function of $\theta$ for $\omega_{b}(\theta=0) / \Delta_{0}=1$. Here $\xi_{i}=\xi_{i}(B, \theta) / \xi_{i}^{0}$ and $\xi_{i}^{0}$ 's are the corresponding coherence lengths for $\varepsilon_{0}=0$ and $B=0$. All these coefficients in the phason propagator develop a dip at the Lebed resonance.

As we shall see this common behavior of the coherence length together with a missing factor in the early theory produces indeed a peak in the electric conductivity $\sigma_{a a}$ at the Lebed resonance $\left(\theta \cong 30^{\circ}\right)$. Also this behavior is independent of the field strength and only requires the proximity of the FISDW transition temperature. If we retained higher order terms in $\left(t_{b} / t_{a}\right)$ in the phase factor of the Green function [19], we would obtain similar resonances at $\tan \theta= \pm p b / c$ with $p$ integer. On the other hand resonances at $\tan \theta= \pm$ $b / q c$ with integer $q$ should be much weaker since these involves the higher order terms in $\left(t_{c} t_{a}\right)$.

\section{Torque and Magnetoresistance.}

As already discussed by Bjeliš and Maki [10], the SDW fluctuation contributes to both thermodynamic quantities like torque and transport coefficient like electric conductivity in the chain direction. In particular the free energy and torque in the 3D system are given by [10]

$F=-\frac{T}{3 \pi \xi_{a} \xi_{b} \xi_{c}}(\eta)^{\frac{3}{2}}$

and

$\tau=\frac{T}{3 \pi} \frac{\partial}{\partial \theta}\left[\left(\xi_{a} \xi_{b} \xi_{c}\right)^{-1}(\eta)^{\frac{3}{2}}\right]$

Note that the present expression is twice larger than the one given in [10], since we now taken care of two fluctuation modes related to two transverse spin components to the external magnetic field [20]. Both free energy and torque are evaluated for $T / T_{c 0}=0.677,0.60$ and 0.55 and shown in Fig. 3 as function of $\theta$ for $\omega_{b}$ $(\theta=0)=\Delta_{0}$. Here $\tilde{F}$ and $\tilde{\tau}$ are $F$ and $\tau$ normalized by $T_{c} \xi_{a}{ }^{o} \xi_{b}{ }^{o} \xi_{c}{ }^{o} \cong 10^{-21} T_{c} / c c$. We see a large dip in free energy and a typical shape of torque at the resonance, since the maximal value of $\tau$ is about $10^{3}$ /radian this large effect should be readily observable. Also the effect becomes stronger as $T_{c}$ is approached.

The electric conductivity in the chain direction is given by Eq (1), where we have already taken care of the above factor two. In Fig. 4 we show the coefficient $\sum=\alpha \tilde{\xi}_{a}\left(\tilde{\xi}_{b} \tilde{\xi}_{c}\right)^{-1}$ which controls the conductivity as function of $\theta$ for $\omega_{b}(\theta=0) / \Delta_{0}=1$. As expected $\sum$ develops a dip at the Lebed resonance, which gives rise to a peak in the magnetoconductance.

\section{Concluding Remarks.}

Considering the size of the resonance effect in magnetoresistance of some of Bechgaard salts, we conclude that this should be either due to scattering effect [8] or the SDW fiuctuation [10]. While the former process give peaks in the magnetoresistance in sharp contrast to experiments [2 4], the present analysis shows that the latter process give indeed dips at the resonance in accordance with experiments. Therefore we conclude 
that the SDW fluctuation is the origin of the Lebed resonance. In order to make a quantitative comparison, it is necessary to develop a theory at much lower magnetic field. We discover also the nature of the Lebed state (i.e. the FISDW at the Lebed resonance) is the doubly quantized state with quantum Hall effect both in the $a-b$ plane and the $a-c$ plane. It should be of great importance to test the present prediction, since the doubly quantized Hall effect has not been seen before.

\section{Acknowledgements.}

We benefitted from discussions with D. Baeriswyl and A. Bjelis on SDW fluctuations. The present work is supported by National Science Foundation under Grant No. DMR 92-18317.

\section{References}

[1] LEBED A.G., Pis'ma Žh. Eksp. Teor. Fiz.43 (1986) 137; JETP Lett 43 (1986) 174.

[2] OSADA T., KAWASUMI A., KAGOSHIMA S., MIURA M. and SAITO G., Phys. Rev. Lett. 66 (1991) 1525.

[3] NAUGHTON M.J., CHUNG O.H., CHAPARALA M., BU X. and COPPENS D., Phys. Rev. Lett.67 (1991) 9721.

[4] KANG W., HANNAHS S.T. and CHAIKIN P.M., Phys. Rev. Lett. 69 (1992) 2827.

[5] MURATA K., BAND H., KAIIMURA K., ISHIGURO T. and ANZAI H., Mol. Cryst. Liqui. Cryst. 119 (1985) 131.

[6] BOEBINGER G.S. et al, Phys. Rev. Lett. 64 (1990) 591.

[7] CHEN L. and MAKI K., Synth. Metals 29 (1989) F493.

[8] LEBED A.G. and BAK P., Phys. Rev. Lett. 63 (1989) 1315.

[9] YAKOVENKO V.M., Phys. Rev. Lett. 68 (1992) 3607.

[10] BJELIŠ A. and MAKI K., Phys. Rev.B 44 (1991) 6791; 45 (1992) 12887.

[11] MAKI K., Phys. Rev. B 45 (1992) 5111.

[12] OSADA T., KAGOSHIMA S. and MIURA M., Phys. Rev. B 46 (1992) 1812.

[13] YAKOVENKO V.M., Phys. Rev. B 43 (1991) 11353.

[14] YAMAJI K.J., Phys. Soc. Jpn 51 (1982) 2787; 52 (1983) 1361.

[15] ISHIGURO T. and YAMAJI K., “Organic Superconductors” (Springer-Berlin 1990).

[16] YAMAJI K., J. Phys. Soc. Jpn 54 (1985) 1034; Synth. Metals 13 (1986) 29.

[17] VIROSZTEK A., CHEN L. and MAKI K., Phys. Rev. B 34 (1986) 3371.

[18] POILBLANC D., HÉRITIER M., MONTAMBAUX G. and LEDERER P., J. Phys. C 19 (1986) L321.

[19] MAKI K., Synth Metals 55 (1993) 2808.

[20] BJELIŠ A. and ZANCHI D. (private communication).

[21] MAKI K. and SUN Y., Prog. Theor. Phys. Supplement (1993). 